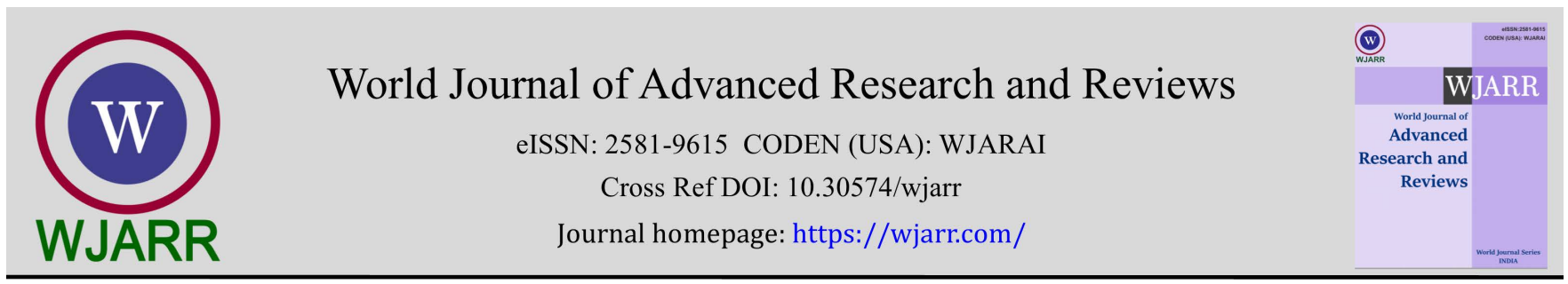

(RESEARCH ARTICLE)

Check for updates

\title{
Music, dance and painting to treat eating disorders; The Chinese TIBET-Model
}

\author{
Wolfgang Mastnak* \\ Beijing Normal University BNU.
}

World Journal of Advanced Research and Reviews, 2022, 13(01), 590-598

Publication history: Received on 17 December 2021; revised on 19 January 2022; accepted on 21 January 2022

Article DOI: https://doi.org/10.30574/wjarr.2022.13.1.0066

\begin{abstract}
Eating disorders are considered an increasing public health problem, also in China. They comprise a broad spectrum of medical conditions such as anorexia nervosa, avoidant/restrictive food intake disorder, bulimia and binge eating, which often go hand in hand with considerable mental and physiological issues such as obesity and metabolic syndrome. Scientific research and clinical experience suggest distinct therapeutic interventions; many of them are related to cognitive behavioural therapy and psychoeducation. The present article introduces artistic approaches comprising music, dance and painting, the so called TIBET-Model, which is based on five principles, namely trance, identity, beauty, exploration and transition, hence the term. This model can be applied as add-on therapy, complementary therapy or main therapeutic intervention, depending on the patient's attitude toward creative and aesthetic processes, as well as therapeutic responses.
\end{abstract}

Keywords: Anorexia nervosa; Bulimia; Chinese arts therapies; Health education; Resource orientation

\section{Introduction}

Eating habits depend on physiological conditions, sociocultural traditions and individual attitudes, hence their complex anthropological characteristics alongside the epistemological problem with the notion 'eating disorder' (ED). In Europe the history of eating disorders goes way back to the Middle Ages - and analyses of the phenomenon reveal how our understanding of abnormal eating has been changing over the centuries.

Examples of self-starvation appeared already in the Hellenistic era, and since the $12^{\text {th }}$ and $13^{\text {th }}$ centuries this form of self-harming has been reported as a religious act [1]. Even today's psychiatry is confronted with cases of anorexia nervosa driven by the idea of religious sacrifice [2]. In the $17^{\text {th }}$ and $18^{\text {th }}$ century, the Age of Enlightenment crucially changed philosophical views and sciences gained in importance, medical domains included. In 1689 Richard Morton [3] gave the first medical description of anorectic symptoms and the English physician Sir William Gull (1816-1890) coined the name 'anorexia nervosa' (AN). When in 1903 Pierre Janet published a detailed description of patients with bulimia [4], the polarity of eating disorders - shortly, too little or too much - was delineated and the way to a modern understanding of eating disorders alongside their modes of treatment paved [5].

While in the early 1900s physicians tried to explore eating disorders as a medical condition of the endocrine system, three decades later these pathological occurrences were rather taken as psychological issues - and in 1973 Hilde Bruch published her book with case studies on eating disorders, a cornerstone of clinical research in this area [6]. Research on abnormal eating behaviour was flourishing and in 1980 eating disorders became a diagnosable mental health condition: they were added to the Diagnostic and Statistical Manual of Mental Disorders (DSM). Also due to epidemiological dynamics of abnormal eating behaviour, binge eating was recognised as a distinct diagnosis by the DSM-5 in 2013 and clearly distinguished from anorexia and bulimia. Nowadays, this medical condition is regarded as the most prevalent

\footnotetext{
* Corresponding author: Wolfgang Mastnak

Beijing Normal University BNU.

Copyright (C) 2022 Author(s) retain the copyright of this article. This article is published under the terms of the Creative Commons Attribution Liscense 4.0.
} 
type of eating disorders and goes hand in hand with obesity, which has become a serious paediatric challenge in modern China.

Although 10 years ago an epidemiological study [7] considered eating disorders a relatively rare condition among the general population, it still stated that 'all eating disorders have an elevated mortality risk; anorexia nervosa the most striking. Compared with the other eating disorders, binge eating disorder is more common among males and older individuals'. By contrast, a recent review [8] speaks of high prevalence and concluded that 'despite the complexity of integrating all ED prevalence data, the most recent studies confirm that EDs are highly prevalent worldwide, especially in women. Moreover, the weighted means of point ED prevalence increased over the study period from 3.5\% for the 2000-2006 period to $7.8 \%$ for the 2013-2018 period. This highlights a real challenge for public health and healthcare providers'.

Eating disorders are dangerous and results of a Chinese cross-sectional study [9] including a sample of 8,746 Chinese adolescents from 20 middle schools in seven provinces - Hebei and Inner Mongolia in the north, Shandong in the east, Heilongjiang in the northeast, Guangxi in the south, Sichuan in the southwest and Shaanxi in the northwest of China indicated 'that all levels of anorexia nervosa serve as predictable indicators of suicidal thoughts in Chinese adolescents'. While anorexia nervosa, self-starvation and the relatively new diagnosis of avoidant/restrictive food intake disorder (ARFID) [10] represent one pole of abnormal eating behaviour, binge eating, which may go hand in hand with obesity, is the other. And according to a comprehensive review study [11] overweight is becoming a serious problem in Chinese paediatrics, particularly in child and adolescent psychiatry.

\begin{abstract}
This meta-analysis of overweight and obesity (ow/ob) among children and adolescents in China from 1991 to 2015 provides a reference for promoting the healthy development of children and adolescents. The studies were retrieved from the China National Knowledge Infrastructure, Wanfang, and PubMed databases for the period from January 1991 to January 2018. The data were combined and analyzed, and the combined effect magnitude odds ratio and the $95 \%$ confidence interval were calculated. Publication bias was determined using Stata/SE12.0. We found that (1) the prevalence of ow/ob increased from $5.0 \%$ and $1.7 \%$ in $1991-1995$ to $11.7 \%$ and $6.8 \%$ in 2011-2015, respectively, and the overweight rate was the greatest in 20062010; (2) from 1991 to 2015, the prevalence of ow/ob was greater in urban areas than in rural areas; (3) compared with girls, boys were more likely to be ow/ob; and (4) the prevalence rates of ow and ob were greater in infancy than in other growth stages, with values of $11.7 \%$ and $7.0 \%$, respectively. The prevalence of ow/ob among Chinese children and adolescents showed significant differences based on region, sex, and age. An overall upward trend was observed that decreased slightly from 2011 to 2015 .
\end{abstract}

According to cross-cultural psychiatry and culturally sensitive music therapy we also have to take cultural differences into consideration. In this context, a comparison of China, Spain and the United Kingdom [12] showed that patients from Western societies shared more similarities regarding psychopathological expression of anorexia nervosa. Moreover, the authors said that while Western countries showed higher levels of body dissatisfaction, somatisation and overall psychopathology, Chinese patients tended to deny or minimise depression, anxiety and other psychopathological symptoms: 'In conclusion, our findings suggest that psychopathological expression of AN is better explained by Western/Eastern influence than by individualist/collectivist values. Although the diagnosis for the eating disorder may be the same, differences in the psychopathology comorbid to the eating disorders may suggest the need for treatments to be modified according to the culture' - and this view coincides precisely with the standpoint of the present article.

Eating disorders are commonly treated with multifactorial psychotherapy including cognitive-behavioural, educational and physical health approaches. Curative plans usually regard the union of mind, body and behaviour, while former methods that focused on only one of these elements made treatment of eating disorders less effective. Efficient treatment requires a clear diagnosis and an evidence-based selection of appropriate interventions, such as recommended in the NICE guideline NG69 - NICE is the abbreviation of National Institute for Health and Care Excellence in the UK - for the recognition and treatment of eating disorders, last updated on the $16^{\text {th }}$ of December 2020 [13]:

For adults with anorexia nervosa, consider one of: individual eating-disorder-focused cognitive behavioural therapy (CBT-ED); Maudsley Anorexia Nervosa Treatment for Adults (MANTRA); specialist supportive clinical management (SSCM) [...] Consider anorexia-nervosa-focused family therapy for children and young people (FT-AN), delivered as single-family therapy or a combination of single- and multi-family therapy. Give children and young people the option to have some singlefamily sessions [...] If FT-AN is unacceptable, contraindicated or ineffective for children or young people with anorexia nervosa, consider individual CBT-ED or adolescent-focused psychotherapy for anorexia nervosa (AFP-AN) [...] Explain to people with binge eating disorder that psychological treatments aimed at treating binge eating have a limited effect on body weight and that weight loss is not a therapy target in itself [...] If guided self-help is unacceptable, contraindicated, or ineffective after 4 weeks, offer group eating-disorder-focused cognitive behavioural therapy (CBT-ED) [...] For children and young people with binge eating disorder, offer the same treatments recommended for adults with binge eating disorder [...] Offer bulimia-nervosa-focused family therapy (FT-BN) to children and young people with bulimia nervosa [...] If FT-BN is 
unacceptable, contraindicated or ineffective, consider individual eating-disorder-focused cognitive behavioural therapy (CBT-ED) for children and young people with bulimia nervosa.

The British approach strongly recommends to take both evidence-based treatment and individual conditions into consideration. This is entirely in line with this article's position, which additionally recommends to focus on sociocultural impacts on eating disorders and to critically discuss the notion 'evidence based' from an epistemological perspective.

\section{Material and methods}

The TIBET-Model was developed through constructive research using key elements of systemic meta-syntheses. The method of systemic meta-syntheses [14] was designed to construct new theories and to generate 'powered hypotheses', a process which can be considered complementary to the estimation of effect sizes as well as hypothesis-testing. In short: the various concepts of verification, power and truth matter.

Akin to the concept of degrees of corroboration, the notion 'powered hypothesis' is based on epistemological strength: (i) similar to meta-analyses systemic meta-syntheses carefully select their components; different from meta-analyses, however, they are chosen because of their epistemological value and trans-disciplinary 'goodness of fit'; (ii) while the weight of a meta-analysis stems from the amount and methodological quality of the studies referred to, meta-syntheses depend on the quality of their linkage and how this blends with the inner logic of the entire framework; (iii) in line with the gestalt-theoretical argument that the whole is greater than the sum of its parts, the theory of systemic metasyntheses argues that the outcome of the whole study in not a simple and direct consequence of their parts, but substantially involves the mode of connecting its components and the epistemological rationale behind the synthesis, hence the term 'powered hypothesis', which also applies to the present study.

\section{Results and discussion}

The TIBET-model consists of artistic practice and therapeutic principles. The first area comprises basically music, dance and painting, but may also include other activities such as poem writing or creative video arts. The second encompasses trance processes, mindful dealing with self- and body-identities, profound experience of beauty related to bodily expression and the embodied self, exploration of body-self and self-images, as well as artistic transitions which are considered a creative way of coping with pathogenic factors.

\subsection{Trance}

Although hypnotherapy is not commonly considered a first-line treatment of eating disorders, a German study [15] discussed its effects on various eating disorders such as bulimia nervosa, anorexia nervosa and obesity. Today, hypnotherapy for eating disorders encompasses various specific approaches such as cognitive hypnotherapy to treat bulimia [16]. Abbreviating trance, 'T' is also the first letter of the model's name 'TIBET'.

Performing the TIBET-model, music and dance are also used (i) to induce trance, (ii) as the core medium of hypnotic processes and (iii) to enhance self-actualisation. In Chinese, hypnosis is usually translated with 催眠 cuīmián, which literally means 'to promote sleep'. In this article, however, we rather refer to the notion 恍惚 huănghū, which is usually translated with 'absent minded' or 'dazzled', but evokes the Daoist idea of 恍惚之美 huănghūzhīměi, which can be understood as the beauty of trance as well as of sensory experience through altered consciousness. Moreover, China has a certain tradition of trance dance, such as the 'Witch's Trance-Dance', 跳神 Tiàoshen, literally 'spiritual jumping' or 'divine jumping', described by Pu Songling in his famous book 'Strange Tales from a Chinese Studio', 聊斋志异 liáozhāizhìyì from 1740 [17], which also involves the issue of body and identity [18].

By way of illustration, Dunhuang dance 敦煌舞 Dūnhuángwǔ combines traditional Chinese culture and modern dance art. It draws inspiration from the body movements in the Dunhuang grotto frescoes found in Gansu province in West China. Repetitive movements inspired by Dunhuang dance may facilitate self-hypnotic induction as well as a feeling of identity with one's movement. Broadly speaking, modern hypnotherapy does not trigger trance in order to inhibit bad habits by hypnotic commands, but rather encourages trance-specific creative capacities of endogenous behaviour modification. In this regard, dance-trance can become a key to overcome eating disorders, while the discovery of underlying mechanisms still requires research. 
For instance, people with binge eating disorder (BED) are faced with the challenge how to stop a binge before it happens. In a sense, binge eating is akin to obsessive-compulsive and impulse-control issues, and according to neurocognitive research [19] BED 'appears to be associated with motor disinhibition and impaired executive planning even controlling for obesity'. And although the neurobiological basis of binge-eating disorder is still unclear, obesity in itself has been linked with cognitive dysfunction: 'we compared cognition between people with binge-eating disorder and controls, matched for BMI and other measures. Binge-eating disorder was associated with impaired response inhibition and executive planning. These results inform neurobiological models of binge-eating disorder and may suggest new treatment targets for this condition'. In this context, pre-trained dance movements may help to control the urge to binge eat. Related therapeutic steps may include (i) imagination techniques, (ii) behavioural training and (iii) mindful development of individually mesmerising dance movements.

\subsection{Identity}

There is broad consensus that in many cases eating disorders are interconnected with identity issues that greatly impact on the efficacy of treatment, such as in individuals with anorexia nervosa [20]:

Three meta-themes were generated with the following key findings: grappling with identity, where collaborative and tailored interventions were positively experienced; the quality of the therapeutic relationship, which existed in a recursive relationship; and, rebuilding identity that included therapists standing with the person in recovering a sense of identity outside the anorexic identity. Importantly, interventions that failed to be negotiated with the person were experienced as disempowering however, where a two-way trust existed in the therapeutic relationship, it critically empowered and shaped participants' sense of identity, and broadened the perception that they were valuable as a person.

There is evidence that in patients with eating disorders identity and integrative functions of the self are imbalanced. Related conceptualisations indicate that individuals with an eating disorder are not able to discriminate between the sensations pertaining their body and their emotions [21] or that their experience of their body is not integrated into their self [22]:

Even though patients with AN do not perceive their body as sharply extraneous, as sometimes happens in schizophrenia [...] they do appear to maintain an attitude of "objectification" toward their body, as if their body does not pertain to their self. The body is no longer experienced in a subjective way as "my" body, and thus as personal or self-related. Instead, the body is a mere object that is impersonal or non-self-related, with no special relationship to the self [...] This is consistent with theories that suggest that physiological responses in the body are the origin of emotions [...] Hence, those with anorexia nervosa may not perceive the physiological correlates of the body, and thus they may not experience, identify and express related emotions. A pervasive weakness of the integrative functions of the self may explain why certain personality traits that are common to AN become rigid, such as high harm avoidance, low self-directedness [...] and perfectionism [...] These traits become stable during adolescence, play a key role in the maintenance of AN, and often remain even after recovery from the disorder.

Regarding these pathogenic and pathological moments, art therapy comes into play. The Schön Klinik Roseneck in Bavaria (Germany) is a renowned psychosomatic hospital, which specialises, alongside other psychiatric diagnoses, in the complex treatment of eating disorders. In group art therapy, patients with anorexia nervosa copy the body contour of other AN-patients on big cartridge papers. Each patient is thereafter invited to artistically work on the own silhouette. This process not only provides the possibility to experience the real size of the body, but also to express in a symbolic way desires or fears, or the subjective recognition of external control and inner petrification. This meaningful creative approach may also give rise to a new feeling of identity.

Identity is also a topic of vocal therapy and dance therapy in eating disorders. According to our clinical experiences, selfidentity and self-images of patients with eating disorders, particularly with anorexia nervosa and avoidant/restrictive food intake disorder, tend to freeze, while artistic work with body-movement or vocal expression may evoke the revival of a dynamic and positive identity.

Moreover, body-identity can be profoundly linked with sport-identity [23] - in the context of this article mainly with dance and martial arts such as Tai Chi 太极拳 or Qigong 气功 - and contribute to weight control in obesity as well as the development of muscle mass. Nonetheless, in this area we also have to be aware of distinct sport-related risks of anorexia such as in dancers [24], pre-professional ballet dancers [25] included - and the problem of perfectionism [26] comes afresh into play. 


\subsection{Beauty}

The subjective conception of beauty plays often a crucial role in eating disorders, particularly in anorexia nervosa, and importantly relates to body shapes. Interdisciplinary analyses [27] show that disordered eating attitudes are rapidly increasing, especially among young women in their twenties, and that 'these disordered behaviours result from the interaction of several factors, including beauty ideals. A significant factor is social media, by which the unrealistic beauty ideals are popularized and may lead to these behaviours'. These problems are inextricably intertwined with the obsession of the perfect body, as well as body dysmorphia [28] and body image distortion [29].

While such beauty ideals may control and dominate bodily self-images, artistic approaches can serve as 'concurring' stimuli with therapeutic effect. Dance and dance therapy have become a viable means to improve body image and body self in patients with various diseases [30], also in individuals with eating disorders such as obese patients [31]: 'Obesity and disturbed eating behaviors are both associated with low self-esteem and distorted body images [...] obese patients enrolled in the DTW [dance therapy workshop] displayed a significant improvement in health-related quality of life [...], body consciousness [...] and mental representations linked to self-body image'. Such experiences also involve aesthetic features.

In this context we recommend clinical singing lessons. The opera singer and vocal therapist Teresa Boning [32] developed vocal coaching in psychiatric settings, which considerably differs from usual music therapeutic approaches: she provided singing classes at high artistic and technical levels for psychiatric in-patients. Although vocal progress and artistic performance were main objectives of this intervention, qualitative analyses showed high therapeutic 'side effects'. Her concept differs sharply from models such as expressive therapy: in Boning's method artistic quality matters. However, she explicitly focuses on the individual beauty of a patient's voice and avoids rigid aesthetic criteria, which are likely to result in external assessment and obsessive-compulsive perfectionism - and this is evidently counterproductive. In short: the experienced beauty of the own voice can help to overcome the dominance of body ideals and even encourage new nutrition regimes as well as physical exercise to enhance vocal progress.

\subsection{Exploration}

The TIBET-model suggests a triple exploratory approach: the first concerning a realistic discovery of the own body, the second encouraging profound examination of bodily symbolisation, and the third using the body as a means to discover unconscious features of one's driving forces and personality traits.

In this context, body-shape painting as described in the section about identity comes afresh into play. It makes, for instance, a difference whether the inner area of the body silhouette remains empty while the periphery is full of life, or the room within the body contours is brimming with silent cries. Such creative and aesthetics processes facilitate the dynamic complementarity between expression of inner processes on the one hand and the discovery of hidden feelings, traumata and desires on the other.

Dance can likewise serve as a highly individual way of self-discovery. Although dance-therapy covers a relatively broad spectrum of concepts including 'authentic movement', movement-analytic approaches or psychodynamic models, the German book 'Tanz der Anorexie' [Dance of anorexia] [33] touches upon the problem that some western schools of dance therapy do not provide models tailored to specific diagnoses, but rather stick to their school-immanent principles. This criticism even relates to elaborated approaches such as Capello's BASCICS-model [Body Attitude-SelfhoodCommunication-Interpersonal Dynamics] [34], which is considered a part of dance movement therapy (DMT). There is a certain tendency to generalisation, hence the lack of models explicitly designed for distinct diagnoses such as avoidant/restrictive food intake disorder, bulimia or binge eating.

Chinese dance traditions can be traced back to about 4,000 years BC - and ethnic minorities living in China are brimming with intermodal arts. In the context of the present article we do not recommend selected dances, but encourage focused analyses of traditional folk dances to discover possible benefits for self-exploration. By way of illustration, the sword dance, 剑舞 jiànwǔ, was initially designed for training soldiers before later turning into an acrobatic dance, which is also linked to Tai Chi practices. Of course, patients will (probably) not perform a perfect Chinese sword dance, while movement improvisation with a prop sword may give rise to the experience of control, strength, aggression, imagination of self-harm, as well as exteriorisation versus energetic centring and grounding. It goes without saying that such dancetherapeutic processes do not follow operationalised sequences of patterns, but call for self-discovery through creative authentic movement. 
While exploratory dance to treat eating disorders is - in a sense - an extroverted approach, Sound Focusing [35], one of the four areas of Sound Work [36], which is a comprehensive body-voice-therapeutic concept, works with introspective practices, partly akin to sound meditation in various ways of Zen Buddhism.

Here, the practitioner (or patient) tries to find a vocal sound that is entirely 'his or her sound': it gives a feeling of identity and aesthetic authenticity, although these notions require individual interpretation and cannot be (easily) defined or operationalised. Once decided which sound is most appropriate, the practitioner focuses on his belly region and tries to sense the sound in the area where some Yoga traditions circumscribe the sacral chakra or several Chinese schools of thought localise the origin of Qi. However, these views do not belong to the theoretical framework of Sound Work and TIBET voice-guided body experience.

Through intuitive sensation one may also assign other features to the sound: colours, dynamic shapes or even emotions. In this way, a patient may perceive that below his navel the sound looks purple, is pear shaped or feels like being caressed by good ghosts. Thereafter, the mental sound-focus moves slowly to the heart, where the multifaceted sound sensation may change. The following sound-body journey continues via the throat, the head and the cranial vault to the neck - and splits in two directions, the right and the left shoulder region. It continues over the arms into the palms and returns to the trapezius muscle. From there it continues to the lower back and the pelvis, the gluteus muscles, the legs and the sole of the feet - and back over the front of the legs to the starting point. This highly sensitive body-sound journey may facilitate access to a broad spectrum of body-self features, hence its exploratory efficacy.

\subsection{Transition}

Dealing with mental traumatisation and pathological behaviour are core issues in psychotherapy and psychiatry, and different theoretical approaches have brought about different methods. For instance, psychoanalysis offers classical and newer ways of trauma processing [37], while recent psychodynamic research also shed light on involuntary coping mechanisms [38] - and cognitive behavioural therapy tries to modify pathological cognition such as negative core beliefs and dysfunctional modes of interpretation.

Notwithstanding the significant clinical effects of such approaches, there is still the epistemological question how the mind overcomes adverse experiences and attitudes, and novel approaches and theoretical frameworks enrich the scene, such as the Trauma Resilience and Recovery Program (TRRP) [39] for younger sufferers, which also comprises symptom self-monitoring, an evidence-based approach to monitoring emotional recovery following a stressor:

Approximately 225,000 children [in the USA] sustain injuries requiring hospitalization annually. Posttraumatic stress disorder (PTSD) and depression are prevalent among pediatric patients and caregivers post-injury. Most U.S. trauma centers do not address patients' mental health needs. Better models of care are needed to address emotional recovery [...] TRRP is designed to (a) provide in-hospital education about post-injury emotional recovery and assess child and caregiver distress; (b) track mental health symptoms via a 30-day text-messaging program; (c) complete 30-day PTSD and depression phone screens; and (d) provide evidence-based treatment via telehealth or in-person services or referrals, if needed [...] In hospital, 68.5\% of caregivers and $78.3 \%$ of children reported clinically significant distress levels [...] Our intervention model was feasible and increased reach to families who needed services. Efforts to improve follow-up engagement are discussed, as are initial successes in implementing this model in other pediatric trauma centers.

Although such programmes provide well elaborated interventions and therapeutic structures, there is still the question how trauma processing works. Moreover: What are the underlying mechanisms of resilience and how shall we deal with maladaptive schemas and associated pathological behaviour such as syndromes of eating disorders? In the realm of arts therapies, the principle of intermodal transformation - as described in Intermodal Expressive Therapy - is akin to the TIBET-concept of trauma processing and modification of pathological behaviour [40]:

Intermodal expressive arts therapy involves a trained therapist facilitating the use of more than one modality in succession with people that would benefit from therapeutic change [...] The act of shifting from one art form to another within the therapeutic space, often referred to as intermodal transfer, can lead to a deeper understanding of psychic material by coming at it from multiple directions - such as through movement, the visual arts, music, and writing [...] Through engaging in the progressive use of different methods of expression, decentering from earthly concerns and anxieties becomes possible [...] As such, a method combining intermodal expressive arts therapy with mindfulness could potentially promote healing by allowing for people living with persistent mental illness to engage in present-focused active expression, allowing for a temporary reprieve from symptoms such as intrusive thoughts and negative self-judgement.

However, transition as a core principle of the TIBET-model not only focuses on 'deeper understanding' - this is what we discussed in the section about exploration - but understands the creative flow from one art form to another as an essential way of trauma processing and modification of traumatic material as well as pathological behaviour. In this 
context we do not speak of 'decentering from earthly concerns and anxieties' but of a re-connection to the nature of being.

This viewpoint is inextricably intertwined with traditional Chinese thought, particularly with key ideas in Daoism: Dao as the principle of permanent transition, while Dao itself is the sole entity with constant stability and inner invariability. From that point of view transition must not be understood as a loss or a threat - it is rather the actualisation of the permanent flow of purification and creation [41]: 'Everything issues from the Dao and ineluctably returns to it; Undifferentiated Unity becomes multiplicity in the movement of the Dao. Life and death are contained in this continuing transformation from Nothing into Something and back to Nothing, but the underlying primordial unity is never lost'.

Therapeutic theories, frameworks and schools of thought inevitably involve assumptions about the reality they refer to, the TIBET-model included. In this context, we do not consider this Daoist maxim an ontological opinion, but a principle that inheres in illness and health, hence the deeper sense of creative transitions.

\section{Conclusion}

This model-generating study is part of a huge Chinese project to improve mental health in children and adolescence and to alleviate syndromes through arts-based interventions in educational areas. The relevant spectrum includes, according to Chinese psychiatric epidemiology and mental public health studies, most prevalent conditions in the Chinese younger generation; in addition to eating disorders and obesity these are attention deficit hyperactivity disorder, oppositional defiant disorder, anxiety disorders, depression, stress and burnout syndromes, and mental disturbances caused by measures to control the COVID-19 pandemic such as social isolation.

Next steps include qualitative exploratory studies, studies to generate standardised models alongside quantitative multicentre studies, as well as feasibility studies, which shall help to provide these educational-therapeutic facilities throughout the People's Republic of China. Moreover, cross-cultural studies shall help to adapt them in different cultures and thus contribute to intermodal culturally sensitive creative and aesthetic therapies.

\section{Compliance with ethical standards}

\section{Acknowledgments}

This research received no specific grant from any funding agency in the public, commercial or not-for-profit sectors.

\section{Disclosure of conflict of interest}

The author declares no conflict of interest.

\section{Statement of ethical approval}

No approval is needed for this type of research.

\section{References}

[1] Hällström T. Self-starvation over 1500 years: the work of God, the devil or weight-control? Lakartidningen. 1999; 96(43): 4648-4653.

[2] Davis AA, Nguyen M. A case study of anorexia nervosa driven by religious sacrifice. Case Reports in Psychiatry. 2014; 512764.

[3] Pearce JM. Richard Morton: origins of anorexia nervosa. European Neurology. 2004; 52(4): 191-192.

[4] Pope HG Jr, Hudson JI, Mialet JP. Bulimia in the late nineteenth century: the observations of Pierre Janet. Psychological Medicine. 1985; 15(4): 739-743.

[5] Marks A. The evolution of our understanding and treatment of eating disorders over the past 50 years. Journal of Clinical Psychology. 2019; 75(8): 1380-1391.

[6] Treasure J, Cardi V. Anorexia Nervosa, theory and treatment: Where are we 35 years on from Hilde Bruch's foundation lecture? European Eating Disorders Review. 2017; 25(3): 139-147. 
[7] Smink FR, van Hoeken D, Hoek HW. Epidemiology of eating disorders: incidence, prevalence and mortality rates. Current Psychiatry Reports. 2012; 14(4): 406-414.

[8] Galmiche M, Déchelotte P, Lambert G, Tavolacci MP. Prevalence of eating disorders over the 2000-2018 period: a systematic literature review. American Journal of Clinical Nutrition. 2019; 109(5): 1402-1413.

[9] Lian Q, Zuo X, Mao Y, Luo S, Zhang S, Tu X, Lou C, Zhou W. Anorexia nervosa, depression and suicidal thoughts among Chinese adolescents: a national school-based cross-sectional study. Environmental Health and Preventive Medicine. 2017; 22(1): 30.

[10] Katzman DK, Norris ML, Zucker N. Avoidant Restrictive Food Intake Disorder. Psychiatric Clinics of North America. 2019; 42(1): 45-57.

[11] Guo Y, Yin X, Wu H, Chai X, Yang X. Trends in overweight and obesity among children and adolescents in China from 1991 to 2015: A Meta-Analysis. International Journal of Environmental Research and Public Health. 2019; 16(23): 4656.

[12] Agüera Z, Brewin N, Chen J, Granero R, Kang Q, Fernandez-Aranda F, Arcelus J. Eating symptomatology and general psychopathology in patients with anorexia nervosa from China, UK and Spain: A cross-cultural study examining the role of social attitudes. PLoS One. 2017; 12(3): e0173781.

[13] NICE. Eating disorders: recognition and treatment.

[14] Mastnak W. Systemic Meta-Synthesis. ResearchGate. 2021.

[15] Barabasz M. Efficacy of hypnotherapy in the treatment of eating disorders. International Journal of Clinical and Experimental Hypnosis. 2007; 55(3): 318-335.

[16] Barabasz M. Cognitive hypnotherapy with bulimia. American Journal of Clinical Hypnosis. 2012; 54(4): 353-364.

[17] Barr A. The textual transmission of Liaozhai zhiyi. Harvard Journal of Asiatic Studies. 1984; 44 (2): 515-562.

[18] Chiang, LSC. Collecting the self: body and identity in strange tale collections of late imperial China (Sinica Leidensia Volume 67). Leiden: Brill. 2005.

[19] Grant JE, Chamberlain SR. Neurocognitive findings in young adults with binge eating disorder. International Journal of Psychiatry in Clinical Practice. 2020; 24(1): 71-76.

[20] Conti JE, Joyce C, Hay P, Meade T. "Finding my own identity": a qualitative metasynthesis of adult anorexia nervosa treatment experiences. BMC Psychol. 22 Oct 2020; 8(1): 110.

[21] Skårderud F. Eating one's words, part I: 'Concretised metaphors' and reflective function in anorexia nervosa - an interview study. European Eating Disorders Review. 2007; 15(3): 163-174.

[22] Amianto F, Northoff G, Abbate Daga G, Fassino S, Tasca GA. Is anorexia nervosa a disorder of the self? A psychological approach. Frontiers in Psychology. 2016; 7: 849.

[23] Mastnak W. Sport identity - Key incentives to adhere to sports-based prevention and rehabilitation. Deutsche Zeitschrift für Sportmedizin [German Journal of Sports Medicine]. 2018; 69(4): 38-44.

[24] Arcelus J, Witcomb GL, Mitchell A. Prevalence of eating disorders amongst dancers: a systemic review and metaanalysis. European Eating Disorders Review. 2014; 22(2): 92-101.

[25] Herbrich L, Pfeiffer E, Lehmkuhl U, Schneider N. Anorexia athletica in pre-professional ballet dancers. Journal of Sports Sciences. 2011; 29(11): 1115-23.

[26] Dahlenburg SC, Gleaves DH, Hutchinson AD. Anorexia nervosa and perfectionism: A meta-analysis. International Journal of Eating Disorders. 2019; 52(3): 219-229.

[27] Aparicio-Martinez P, Perea-Moreno AJ, Martinez-Jimenez MP, Redel-Macías MD, Pagliari C, Vaquero-Abellan M. Social media, thin-ideal, body dissatisfaction and disordered eating attitudes: an exploratory analysis. International Journal of Environmental Research and Public Health. 2019; 16(21): 4177.

[28] Vashi NA. Obsession with perfection: Body dysmorphia. Clinics in Dermatology. 2016; 34(6): 788-791.

[29] Hosseini SA, Padhy RK. Body image distortion. In: StatPearls [Internet]. Treasure Island [FL]: StatPearls Publishing. 2021.

[30] Müller-Pinget S, Golay A. To improve body image with dance therapy. Revue Medicale Suisse. 2019; 15(643): 640-642. 
[31] Muller-Pinget S, Carrard I, Ybarra J, Golay A. Dance therapy improves self-body image among obese patients. Patient Education and Counseling. 2012; 89(3): 525-528.

[32] Boning T. Funktionale Gesangspädagogik im psychiarischen Setting [PhD-dissertation]. Salzburg: University Mozarteum. 2019.

[33] Khvostova O, Willmann M. Tanz der Anorexie. Bewegungs- und Tanztherapie bei Magersucht. Gießen: Psychosozialverlag. 2018.

[34] Capello PP. BASCICS. An intra/interactional model of DMT with the adult psychiatric patient. In: Chaiklin S, Wengrower H, Eds. The Art and Sciences of Dance/Movement Therapy. Life is Dance. New York: Routledge. 2009. 77-102.

[35] Mastnak W. Sound Focusing. Therapie durch Stimme und gezielte Körperresonanz. Musiktherapeutische Umschau. Forschung und Praxis der Musiktherapie. 1992; 13: 30-47.

[36] Mastnak W. Sound Work: Voice and body in psychiatry, psychosomatics and health promotion. Musik-, Tanz- und Kunsttherapie. 2018; 28(1): 109-121.

[37] Levine HB. Trauma, process and representation. International Journal of Psychoanalysis. 2021; 102(4): 794-807.

[38] Vaillant GE. Involuntary coping mechanisms: a psychodynamic perspective. Dialogues in Clinical Neuroscience. 2011; 13(3): 366-370.

[39] Ridings LE, Anton MT, Winkelmann J, Davidson TM, Wray L, Streck CJ, Ruggiero KJ. Trauma resilience and recovery program: addressing mental health in pediatric trauma centers. Journal of Pediatric Psychology. 2019; 44(9): 1046-1056.

[40] Daly M. Expressive mindfulness: a trauma-sensitive expressive arts therapy group method. Expressive Therapies Capstone Theses. 208. 2019.

[41] Basic concepts of Daoism. Encyclopedia Britannica. 